\title{
Treatment of Vaginal Atrophy with the Aqueous Extract of Triticum vulgare: Comparison between Different Pharmaceutical Forms
}

\author{
Mainini $\mathrm{G}^{1 *}$, Ferrentino $\mathrm{A}^{2}$, Lauria $\mathrm{R}^{2}$, Di Donna $\mathrm{MC}^{3}$, Ercolano $\mathrm{S}^{2}$, De Simone $\mathrm{R}^{2}$ and Passaro $\mathrm{M}^{2}$ \\ ${ }^{1}$ Menopause Centre San Leonardo Hospital, ASL NA3 SUD, Italy \\ ${ }^{2}$ ASL NA3 SUD, Italy \\ ${ }^{3}$ Department of Gynecologic Oncology, ARNAS CIVICO, University of Palermo, Italy
}

${ }^{\star}$ Corresponding author: Mainini G, Menopause Centre San Leonardo Hospital, ASL NA3 SUD, Castellammare di Stabia, NA, Italy; Email: giampaolomainini@libero.it

Received: July 29, 2020; Accepted: August 10, 2020; Published: August 14, 2020

\begin{abstract}
Decreased estrogenization in post-menopausal women causes changes in the lower urinary tract. Vulvovaginal Atrophy (VVA) is a pathological condition resulting from those changes. VVA has a negative effect on the quality of life therefore prompting a search for well tolerated therapeutic options. This article was aimed to evaluate whether the participation of the patient in the choice of the therapy may improve the adherence to the treatment. In particular, there are two distinct pharmaceutical forms in vaginal cream and pessaries based on the Triticum vulgare Extract (TVE) for the treatment of post-menopausal VVA. We examined the clinical reports of 48 women with VVA who chose to be treated with Fitostimoline ${ }^{\circledR} 20 \%$ vaginal cream (based on TVE, 2-phenoxyethanol; glycerine; white vaseline; sodium cetyl stearyl sulfate; cetyl stearyl alcohol; decyl oiled; methyl parahydroxybenzoate; propyl parahydroxybenzoate; purified water. Produced by Farmaceutici Damor, Naples, Italy) and 43 who preferred Fitostimoline ${ }^{\circledR} 600$ mg pessaries (based on TVE, 2-phenoxyethanol; macrogol 400; macrogol 1500; macrogol 12000 produced by Farmaceutici Damor, Naples, Italy) for a 3-month treatment. Signs and symptoms of VVA were available during the baseline and after 12 and 90-day treatment visits.
\end{abstract}

Adhering to the patient's choice allowed for a very high compliance to the prescribed therapy in the whole study population, and we recorded a significant reduction of the Total Symptom Score (TSS) in both women treated with the vaginal cream and in those who underwent pessary therapy, without any difference in TTS and in its single components between the two pharmaceutical formulation groups. Thus, the availability of two distinct formulations of Fitostimoline ${ }^{\circledR}$ pessaries and vaginal cream seems to be particularly useful for the treatment of VVA.

Keywords: Vulvovaginal atrophy; Fitostimoline ${ }^{\circledast}$ pessaries; Fitostimoline vaginal cream

\section{Introduction}

Vulvovaginal Atrophy (VVA) is a common condition associated with decreased estrogenization of the vaginal tissue. It can occur at any time in a woman's life cycle, but it is more frequent in the postmenopausal phase, since it affects around $90 \%$ of postmenopausal women [1]. Other than menopause, VVA may appear during hypoestrogenic conditions, such as lactation, various breast cancer treatments, and use of certain medications. In these cases, VVA may resolve spontaneously when estrogen levels are restored [2]. VVA symptoms are all dependent from decreased estrogenization and include: Dryness, soreness, irritation and dyspareunia with increased urinary frequency, urgency, and urge incontinence [3]. Clinical findings include the presence of pale and dry vulvovaginal mucosa with petechiae. Vaginal rugae disappear, and the cervix may become flush with the vaginal wall. A vaginal $\mathrm{pH}$ of 4.6 or more supports the diagnosis of VVA because the low level of estrogen may decrease the number of lactobacilli thus causing increased $\mathrm{pH}$ of the vagina [4].

Only a quarter of women with VVA seek for treatment [5]. The first- line treatment of VVA is a continuous sexual relationship, using non-hormonal lubricant over the counter vaginal [6]. The systemic and local estrogen administration is recommended for the treatment of VVA in postmenopausal women [7]. Although hormone therapy may alleviate the short and long term complications of menopause, such as hot flashes, night sweats, and VVA, it may increase the risk of breast and endometrial cancer [8]. Because of the adverse effects of estrogen therapy, some women prefer not to use this method for alleviating VVA, and they tend to use non-hormonal medication for this matter [9]. In this regard, it is noteworthy the evidence that a local treatment based on hyaluronic acid is more effective than hormonal therapy in improving VVA symptoms [10]. More recently, it has been showed that the aqueous extract of Triticum vulgare (TVE) in cream [11] and pessaries [12] formulation could reduce sign and symptoms of VVA in postmenopausal women. TVE containing mainly poly/ oligosaccharides' components has been extensively used in different pharmaceutical forms. The Damor Farmaceutici TVE, because of its global patent based on its production process, has unique characteristics that lead to its application in VVA treatment. In fact, 
as recently demonstrated, Damor TVE has a regenerating [13,14], antinflammatory [15] and anti-MMP9 activity [16], and antioxidant activity [17], which play a crucial role in the treatment of VVA. Furthermore, these recent studies suggest that Damor TVE is at least as efficacious as hyaluronic acid in favoring tissue re-epithelization and in contrasting inflammation. For this reason, Damor TVE cream and pessaries formulations (Fitostimoline ${ }^{\bullet}$ vaginal cream and pessaries) are used in the gynecological clinical practice as an alternative to hyaluronic acid in the treatment of VVA.

Since in a long-term treatment it may be useful to involve the patient in the choice of the pharmaceutical formulation to use, in order to improve adherence to the treatment, the availability of two different pharmaceutical formulations based on Damor TVE, may be particularly useful to improve the adherence to the treatment. Thus, we used a large population of women with VVA treated with the vaginal cream or the pessaries of Damor TVE, according to their preference, to evaluate in real word clinical practice whether this "therapeutic agreement" may improve the compliance in a long-term treatment.

\section{Patients and Treatments}

In an Outpatient Gynecological Clinic database we selected 91 women, aging between 18 and 70 years, with evidence of VVA (physiological, pharmacologically induced or surgery-dependent) who had symptoms and objective signs of vaginal atrophy plus amenorrhea for at least 12 months, who chose to receive one of following treatments for a 3 months' period:

- Fitostimoline $20 \%$ vaginal cream (based on TVE, 2-phenoxyethanol; glycerine; white vaseline; sodium cetyl stearyl sulfate; cetyl stearyl alcohol; decyl oiled; methyl parahydroxybenzoate; propyl parahydroxybenzoate; purified water. Produced by Farmaceutici Damor, Naples, Italy).

- Fitostimoline $600 \mathrm{mg}$ pessaries (based on TVE, 2-phenoxyethanol; macrogol 400; macrogol 1500; macrogol 12000. Produced by Farmaceutici Damor, Naples, Italy).

The treatments were locally applied every evening for 3 months. Patients were asked to avoid vaginal sexual intercourse during the entire study period.

We excluded women with other gynecological diseases (in addition to VVA), like the presence of metabolic or endocrine diseases (e.g. uncontrolled diabetes mellitus) or of other local/systemic diseases that could potentially interfere with the study parameters (concomitant treatment with antibiotics/antiseptic agents, steroidal and non-steroidal anti-inflammatory drugs, analgesics (except paracetamol as pain killer)). All patients gave their informed written consent to the use of their clinical data for scientific purposes.

\section{Outcome Measures}

Signs and symptoms of VVA were available during the baseline visit and after 12 and 90 days of treatment. Six subjective symptoms (burning, pain, itching, vaginal dryness, dyspareunia and dysuria) and 5 objective signs (mucous dryness, pale mucosa, thin vaginal folds, mucous fragility, petechiae) of vaginal atrophy were evaluated by a semi-quantitative scale $(0=$ absence of the sign/symptom; $1=$ mild sign/symptom; 2 = moderate sign/symptom; 3 = severe sign/ symptom) expressed for both single signs and symptoms and for their sum (total symptoms score, TSS). Adverse events occurrence and vital signs (blood pressure, heart rate, body temperature) were reported as safety parameters. At the end of the treatment period, a satisfaction opinion was expressed by the patient in terms of excellent, good, to be improved, negative. For the statistical analysis, post hoc simultaneous multiple comparisons were done by Bonferroni's analysis.

\section{Results}

Out of 91 women recruited, 48 were treated with Damor TVE vaginal cream and 43 with the pessaries. The two groups of women were comparable by demographics and clinical characteristics (Table 1). The menopause origin for the majority of women was physiological (Table 2). Only a few women had a pharmacological or surgical menopause (Table 2). However, the origins of the menopause were comparable between the two groups of women. All the patients were completely adherent to the treatment since all completed the 90-day treatment period with a compliance, percentage of the prescribed therapy assumed, above 95\%: 98\% for Fitostimoline ${ }^{\bullet}$ vaginal cream and $95 \%$ for Fitostimoline ${ }^{\bullet}$ pessaries (Figure 1).

By evaluating together patients treated with the vaginal cream and the pessaries, a significant reduction of the TSS was detected both at visit 2 and at visit 3 (Figure 2). When the TSS score was evaluated separately for women treated with Fitostimoline ${ }^{\circ}$ vaginal cream and those treated with Fitostimoline ${ }^{\star}$ pessaries no significant difference in the TSS was detected between the treatment groups (Figure 3). As shown in Figure 3, a significant reduction in the TSS occurred from

Table 1: Patient demographic characteristics.

\begin{tabular}{|l|c|c|c|}
\hline & $\begin{array}{c}\text { Pessaries } \\
(\mathbf{N}=\mathbf{3 4})\end{array}$ & $\begin{array}{c}\text { Cream } \\
\mathbf{( N = 3 8 )}\end{array}$ & p Value \\
\hline Age (years) & $51.4 \pm 11.0$ & $53.2 \pm 10.3$ & NS \\
\hline Menopause (months) & $32.0 \pm 34.5$ & $37.5 \pm 31.7$ & NS \\
\hline
\end{tabular}

Table 2: Menopause origin.

\begin{tabular}{|l|c|c|c|}
\hline & $\begin{array}{c}\text { Pessaries } \\
(\mathrm{N}=34)\end{array}$ & $\begin{array}{c}\text { Cream } \\
(\mathrm{N}=38)\end{array}$ & p Value \\
\hline Physiological (\%) & 93.1 & 89.6 & NS \\
\hline Surgical (\%) & 4.7 & 6.3 & NS \\
\hline Pharmacological (\%) & 2.2 & 4.1 & NS \\
\hline
\end{tabular}

Patients adherence to the treatment

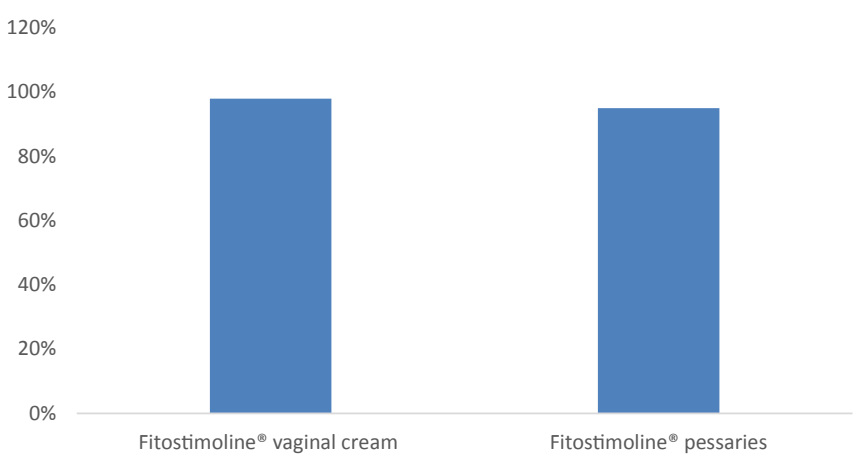

Figure 1: Patients adherence to the treatment with Fitostimoline ${ }^{\varpi}$ vaginal cream and Fitostimoline ${ }^{\varpi}$ pessaries. 


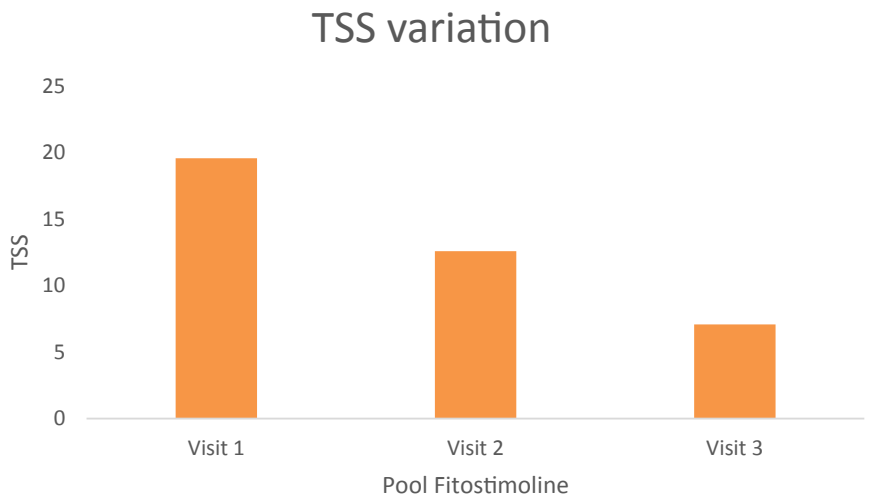

Figure 2: TSS evaluation for treatment with both Fitostimoline ${ }^{\circ}$ pessaries and Fitostimoline ${ }^{\infty}$ vaginal cream.

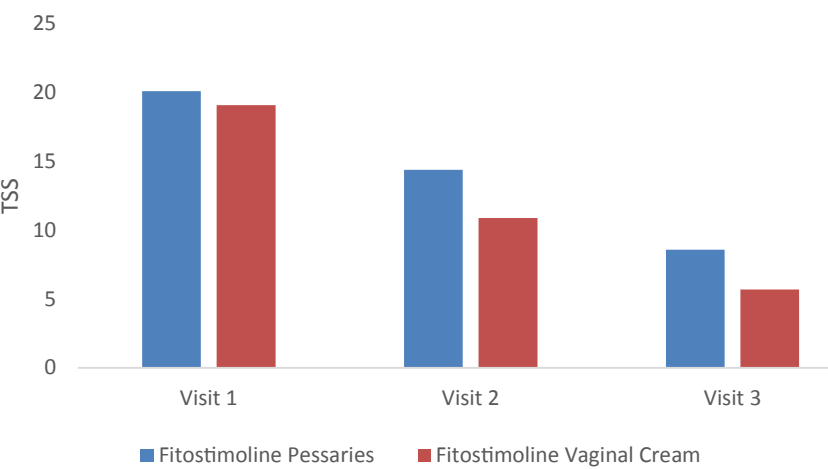

Figure 3: TSS variation in patients treated with Fitostimoline ${ }^{\varpi}$ pessaries and Fitostimoline vaginal cream.

visit 1 to visit 2 in the two study groups (TSS $=20,1$ at visit 1 and 14,4 at visit 2 to for Fitostimoline ${ }^{\oplus}$ pessaries; TSS $=19,1$ at visit 1 and 10,9 at visit 2 for Fitostimoline ${ }^{\bullet}$ vaginal cream). A further significant reduction of the TSS score was observed from visit 2 to visit 3 either in patients treated with the vaginal cream (TSS $=10,9$ at visit 2 and 5,7 at visit 3 ) than in those treated with the pessaries (TSS $=14,4$ at visit 2 and 8,8 at visit 3 ) (Figure 3).

Also the single components of the TSS score of VVA were evaluated for both groups of women (Figures 4 and 5). A significant reduction of the main VVA symptoms of pain, burn, itching, dryness, dyspareunia, dysuria, petechiae was observed both in patients treated with the vaginal cream and those receiving the pessaries at visit 2 and visit 3 versus the basal visit (Figures 4 and 5). Furthermore, a statistically significant progressive improvement of all these symptoms evaluated was observed from visit 2 to visit 3 (Figures 4 and 5) without any difference between the groups of treatment (Figures 4 and 5).

Finally, in order to assess whether the women were satisfied by the chosen treatment, vaginal cream or the pessaries, the patient's satisfaction was evaluated. As indicated by Figure $6,65,1 \%$ of women treated with Fitostimoline ${ }^{\circ}$ pessaries and $68,8 \%$ of women treated with Fitostimoline vaginal cream reported an "excellent satisfaction", $32,6 \%$ and $31,2 \%$, respectively had a "good satisfaction", while only $2.3 \%$ of women treated with Fitostimoline ${ }^{\circledR}$ pessaries referred a "negative satisfaction".

\section{Symptoms variation after treatment with Fitostimoline ${ }^{\circledR}$ pessaries}

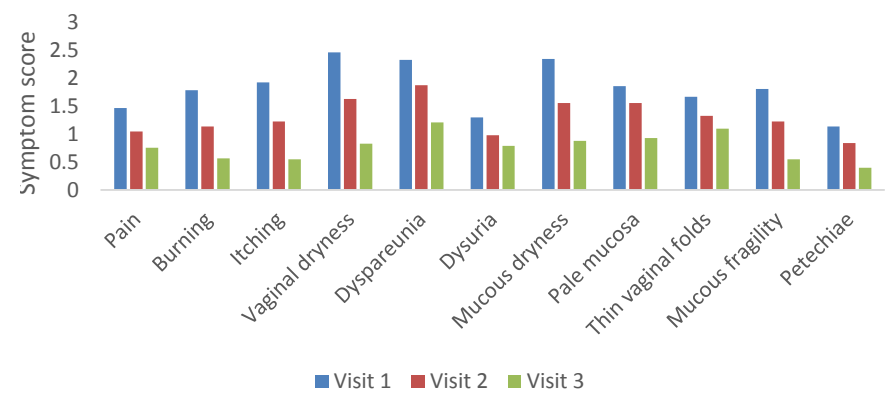

Figure 4: Symptoms variation after treatment with Fitostimoline ${ }^{\infty}$ pessaries.

\section{Symptoms variation after treatment with Fitostimoline ${ }^{\circledR}$ vaginal cream}

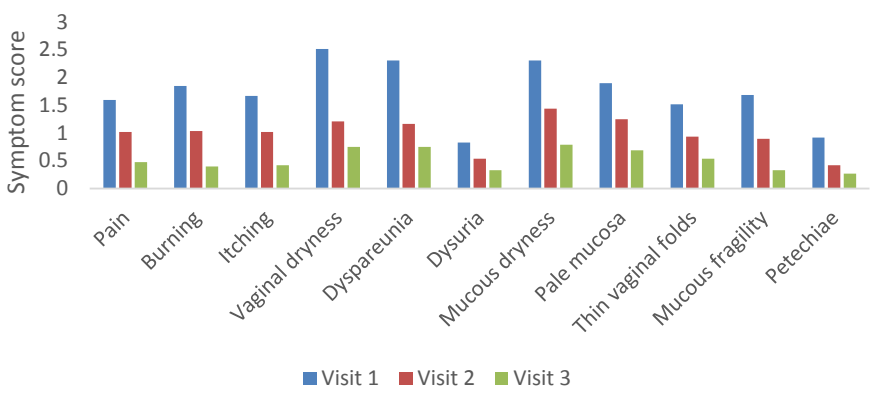

Figure 5: Symptoms variation after treatment with Fitostimoline ${ }^{\infty}$ vaginal cream.

Satisfaction to the treatment

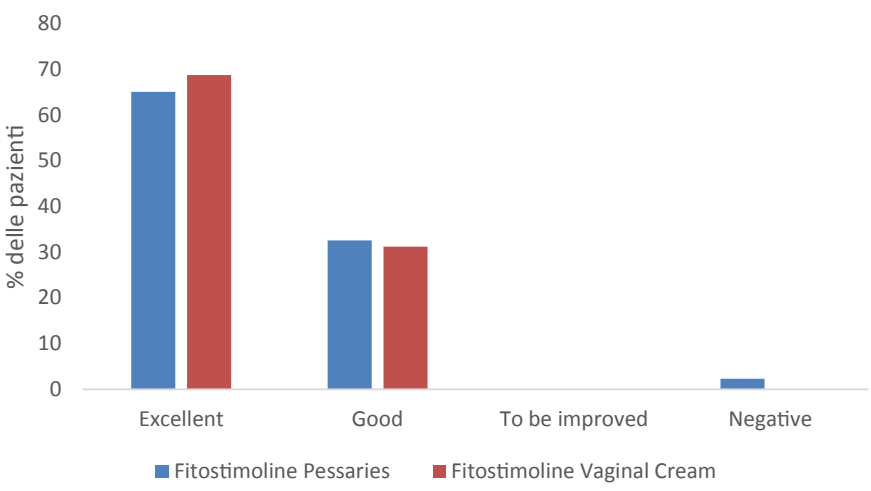

Figure 6: Evaluation of the satisfaction to the treatment with Fitostimoline ${ }^{\varpi}$ pessaries and vaginal cream.

\section{Discussion}

The transition from evidence medicine, "one size fits for all", to precision medicine, "tailored treatment", requires not only the demonstration of the efficacy and tolerability of a given treatment obtained in randomized trials but also its detailed characterization which can be obtained exclusively in daily clinical practice using large groups of patients. Furthermore, in order to solve the problem of the low adherence and compliance to the prescribed therapy observed in all the long-term treatments, it has been suggested that the patient should be adequately informed and involved in the choice of the therapy. 
Thus, the availability of large database of Outpatient Clinics seems particularly useful to satisfy these needs for very common diseases which require long-term treatments. In order to verify this assumption for VVA we obtained from the data base of an Outpatient Gynecological Clinic the clinical records of 91 women with VVA who had been involved in the choice of the treatment and had undergone a 90-day therapy with Fitostimoline ${ }^{\bullet}$ vaginal cream or with Fitostimoline ${ }^{\bullet}$ pessaries. The results of the present study confirm that the involvement of the patients in the choice of the treatment markedly improves the adherence and the compliance to the therapy prescription since at our knowledge there are no reports in the real world clinical practice describing a $100 \%$ adherence in a 3 month treatment with the assumption of more than $95 \%$ of the drug prescribed.

Our results do not allow any speculation of the efficacy of TVE pharmacological formulations in the treatment of VVA since we have no comparison with placebo or active drug. However, experimental studies suggest that there is a rationale for the use of TVE for the treatment of VVA [13-16] and evidence of their efficacy in the treatment of VVA obtained in clinical studies are available in the literature $[11,12]$. An indirect support to these data seems to be corroborated by the comparison between the percentage of success achieved in our population, as expressed by the reduction in TSS score, and that reported with the use of hyaluronic acid in the treatment of VVA (Figure 2) [10].

Furthermore, the observation that there are no differences between TVE pessaries and vaginal cream either in the evaluation of the TSS or in the evaluation of the single symptoms, support the possibility to allow a personal choice between the two pharmacological formulations. Finally, the time course of the therapeutic effect that appears to consolidate over time seems to suggest that the mechanism of action of the Fitostimoline formulations is actually pathogenic rather than symptomatic. The limitations of this study are that this is not a clinical trial but an observational study on a wide range of cases. For this reason, we do not have a validation of efficacy which however already exists for this products and it was not in the objective of the study which consisted in assessing whether the availability of different pharmaceutical forms by favoring the "therapeutic concordance" can be useful in improving compliance.

\section{Conclusion}

For the purposes of therapeutic agreements between physicians and patients, which is the mechanism currently suggested to improve adherence to therapy, the availability of different pharmaceutical forms represents an issue of great relevance. In order to manage the choice by the physician, however, it is essential to have full knowledge of the potential of the different pharmaceutical forms. For this reason, we can conclude that the two distinct formulations of TVE, pessaries and vaginal cream, didn't show any significant difference in the treatment of the VVA, both in terms of efficacy and of compliance, and could be alternatively used for the treatment of VVA.

\section{References}

1. Palacios S, Nappi RE, Bruyniks N, Particco M, Panay N (2018) The European Vulvovaginal Epidemiological Survey (EVES): Prevalence, symptoms and impact of vulvovaginal atrophy of menopause. Climacteric 21: 286-291. [crossref]

2. Mac Bride MB, Rhodes DJ, Shuster LT (2010) Vulvovaginal atrophy. Mayo Clin Proc 85: 87-94. [crossref]

3. Castelo-Branco C, Cancelo MJ (2008) Compounds for the treatment of atropic vaginitis. Expert Opin Ther Pat 18: 1385-1394.

4. Sturdee DW, Panay N (2010) Recommendations for the management of postmenopausal vaginal atrophy. Climacteric 13: 509-522. [crossref]

5. Castelo-Branco C, Rostro F (2007) Treatment of atrophic vaginitis. Therapy 4: 349353.

6. Noumova I, Castelo-Branco C (2018) Current treatment options for postmenopausal vaginal atrophy. Int J Women's Health 10: 387-395 [crossref]

7. North American Menopause Society (2007) The role of local vaginal estrogen for treatment of vaginal atrophy in postmenopausal women: 2007 position statement of the North American Menopause Society. Menopause 14: 355-369. [crossref]

8. Wentzensen N, Trabert B (2015) Hormone therapy: Short-term relief, long-term consequences. Lancet 385 .

9. Ibe C, Simon JA (2010) Vulvovaginal atrophy: Current and future therapies. J Sex Med 7: 1042-1050. [crossref]

10. Jokar A, Davari T, Asadi N, Ahmadi F, Foruhari S (2016) Comparison of the hyaluronic acid vaginal cream and conjugated estrogen used in treatment of vaginal atrophy of menopause women: a randomized controlled clinical trial. Int $J$ Community Based Nurs Midwifery 4: 69-78. [crossref]

11. Mollica G, Bonaccorsi G, Martinello R (2008) Evaluation of efficacy and tolerability of Fitostimoline vaginal cream (Damor Farmaceutici) in the treatment of vaginal inflammation and vulvar dystrophy: A double-blind randomized controlled trial Gazzetta Medica Italiana Archivio per le Scienze Mediche 167: 87-95.

12. Mollica G, Bonaccorsi G, Martinello R (2008) Valutazione di efficacia e tollerabilità di Fitostimoline Ovuli Vaginali (Damor Farmaceutici) nel trattamento delle affezioni flogistico-distrofiche della vagina. Studio controllato in doppio cieco. Gazzetta Medica Italiana Archivio per le Scienze Mediche 167: 97-103

13. Sanguigno L, Minale M, Vannini E, Arato G, Riccio R, et al. (2015) Oligosaccharidic fractions derived from Triticum vulgare extract accelerate tissue repairing processes in in vitro and in vivo models of skin lesions. J Ethnopharmacol 159: 198-208. [crossref]

14. D’Agostino A, Pirozzi AVA, Finamore R, Grieco F, Minale M, et al. (2020) Molecular mechanisms at the basis of pharmaceutical grade triticum vulgare extract efficacy in prompting keratinocytes healing. Molecules 25: 431. [crossref]

15. Sanguigno L, Casamassa A, Funel N, Minale M, Riccio R, et al. (2018)Triticum vulgare extract exerts an anti-inflammatory action in two in vitro models of inflammation in microglial cells. PLoS One. 13: 197493. [crossref]

16. Funel N, Dini V, Janowska A, Loggini V, Minale M, et al. (2020) Triticum vulgare extract modulates protein-kinase $\mathrm{b}$ and matrix metalloproteinases 9 protein expression in bv-2 cells: Bioactivity on inflammatory pathway associated with molecular mechanism wound healing. Mediators of Inflammation 1-13.

17. Antonucci I, Fiorentino G, Contursi P, Minale M, Riccio R, et al. (2018)Antioxidant capacity of rigenase ${ }^{\infty}$, a specific aqueous extract of triticum vulgare. Antioxidants (Basel) 7: 67. [crossref]

\section{Citation:}

Mainini G, Ferrentino A, Lauria R, Di Donna MC, Ercolano S, De Simone R and Passaro M (2020) Treatment of Vaginal Atrophy with the Aqueous Extract of Triticum vulgare: Comparison between Different Pharmaceutical Forms. ARCH Women Health Care Volume 3(3): 1-4. 